\section{Discussion}

Improvements in technique appear to allow endarterectomy to be performed below the inguinal ligament with a reasonable initial success rate. In this series it is $95 \%$. If the occluded segment is of limited length this operation is preferred to the femoro-popliteal bypass graft. In practice, however, the arteriogram often gives an inadequate assessment of the length of artery requiring endarterectomy. This is frequently much longer than is apparent radiologically.

When the superficial femoral artery alone is the site of operation, the common femoral and popliteal arteries must be carefully scrutinized on the arteriograms. If any doubt exists about their adequacy they must be explored at operation, because disease above or below the superficial femoral endarterectomy may cause primary failure. When aorto-iliac occlusive disease is present in addition to disease below the inguinal ligament endarterectomy can be performed simultaneously above and below the inguinal ligament. In short occluded segments we prefer to use direct endarterectomy without stripping. In long occluded segments, if the atheromatous material separates easily, we use a stripper. Arteries are stripped under direct vision to ensure that no damage is done to the outer wall of the vessel, and any serious resistance to stripping requires a further arteriotomy. In all cases, whether stripping is done or not, the lowest limit of the endarterectomy is directly visualized through an arteriotomy, and the distal intima anchored where required.

We believe that any arteriotomy distal to the common femoral artery should be closed with a vein or Dacron patch, and some common femoral arteriotomies also require this. Some surgeons recommend arteriography on the operating-table, after stripping to ensure that no atheromatous fragments are left in the arterial lumen (Wylie, Binkley, and Albo, 1964). We have not practised this. It would certainly appear to be a wise precaution where stripping was difficult, but we prefer open endarterectomy under direct vision in these conditions.

The primary results have been encouraging, and all of those cases presenting with claudication were relieved of their symptoms. In the group of seven patients presenting with gangrene or rest pain the alternative to arterial reconstruction was a major amputation, and all but one were saved this. The last patient would also have kept his limb, because his rest pain was relieved after a successful endarterectomy, had not wound infection reached the arterial suture line and haemorrhage from it necessitated proximal ligation and amputation. Against this salvage of six limbs must be set the loss of one limb in a claudicant where wound infection also required proximal ligation of a successful arterial reconstruction. These two cases emphasize the seriousness of wound infection in arterial operations, and in the limbs such infections almost invariably involve the arterial suture lines.

It remains to be seen whether long-term patency after endarterectomy below the inguinal ligament is superior to that seen after femoro-popliteal bypass graft.

\section{Summary}

The long-term results of cloth bypass grafts below the inguinal ligament have not been encouraging, and the calibre of the patients long saphenous vein is often inadequate. Improvements in technique have led to reintroduction of endarterectomy for superficial femoral and popliteal artery occlusions. This paper reports the results of femoro-popliteal endarterectomy for superficial femoral and popliteal artery formed by the open method or by using an intraluminal stripper, and the importance of using vein or Dacron patches to avoid narrowing the arteriotomy closure has been emphasized. The initial success rate was $94 \%$. The importance of treating coexisting proximal or distal disease was stressed, and the primary and late results have been analysed.

\section{REFERENCES}

Cannon, J. A., Barker, W. F., and Kawakami, I. G. (1958). Surgery, 43, 76.

Edwards, W. S. (1962). Ibid., 51, 58.

Irvine, W. T., Kenyon, J. R., and Stiles, P. J. (1963). Brit. med. F., 1, 360.

Kunlin, J. (1951). Rev. Chir. (Paris), 70, 206

Wylie, E. J., Binkley, F. M., and Albo, R. J. (1964). Amer. F. Surg., 108, 215 .

\title{
Studies with Complamin, a Nicotinic-acid-Theophylline Ester, as a Fibrinolytic Agent
}

\author{
G. P. MCNICOL, ${ }^{*}$ M.D., M.R.C.P.ED., M.R.C.P.GLASG. ; \\ A. S. DOUGLAS, ${ }^{*}$ M.B., B.SC., F.R.C.P., F.R.C.P.GLASG., F.R.C.P.ED.
}

Brit. med. f., 1965, 1, 1149-1153

Though the feasibility of thrombolytic therapy with fibrinolytic enzymes has now been demonstrated-for example, Fletcher et al. (1959)-the expense involved in the preparation of suitable enzymes and the difficulties in laboratory control of enzyme therapy have stimulated a search for non-enzymatic agents which when administered might stimulate production or release of fibrinolytic enzymes in the body. One such substance is nicotinic acid, which, fibrinolytically inert in vitro, was discovered by Meneghini and Piccinini (1958) and Weiner et al. (1958) to have the property of inducing intense but transient fibrinolytic activity when injected intravenously in man. Because of the short duration of the fibrinolytic response and the rapid development of resistance, attempts have been made to provide modified preparations of nicotinic acid with enhanced activity, and it is the purpose of this paper to describe studies with one such preparation 3-(methyloxyethylamino)-2- oxypropyl theophylline nicotinate-Complamin. The manufacturers of this preparation (J. A. Wulfing, Düsseldorf) recommend it in the treatment of a very wide variety of vascular diseases, including acute peripheral vascular occlusion, thrombophlebitis, pulmonary and cerebral embolism, and myocardial infarction. Side-effects are said by the manufacturers to be "quite insignificant," and in particular freedom from side-effects on the circulatory system is claimed.

The fibrinolytic enzyme system is complex, but the main components are plasminogen, plasmin, activators, and inhibitors. Plasminogen, a normally inert plasma globulin, is converted by activators, themselves highly specific proteolytic enzymes, to plasmin, a proteolytic enzyme which under suitable circumstances digests fibrin, with release of soluble products.

* University Department of Medicine, Glasgow Royal Infirmary. 
Activators of plasminogen include tissue activators, a plasma activator, a urinary activator named urokinase, and bacterial activators-for example, streptokinase. Under physiological circumstances, when fibrin is laid down it carries with it substantial amounts of plasminogen, and the physiological mechanism of fibrinolysis, as suggested by Sherry et al. (1959a), probably involves the diffusion of activator from the plasma into fibrin, with activation of intrinsic clot plasminogen; the resulting plasmin is in close association with fibrin, which is lysed-the process of thrombolysis. In plasma, on the other hand, plasmin produced by physiological levels of activator is neutralized by antiplasmin and plasma proteins are protected from digestion.

With high levels of plasminogen activator in the circulationfor example, in certain haemorrhagic states or during thrombolytic therapy-rapid conversion of plasma plasminogen to plasmin results in temporary overwhelming of the antiplasmin mechanism, with the appearance of free plasmin in the circulation-hyperplasminaemia. The consequences of hyperplasminaemia include digestion of coagulation factors, including fibrinogen, factor V, prothrombin, and antihaemophilic globulin, and defective fibrin polymerization due to the presence in the circulation of fibrinogen breakdown products which interfere with the conversion of fibrin monomer to fibrin polymer, and hence prolong the thrombin clotting-time and the one-stage prothrombin time (Fletcher et al., 1964). The object of thrombolytic therapy is to increase plasma activator levels, and hence plasma thrombolytic activity, with the object of removing occlusive thrombus before distal tissue necrosis or other deleterious effects have occurred ; a degree of hyperplasminaemia has usually to be accepted as an inevitable concomitant of a significant increase in activator levels. Nicotinic acid owes its fibrinolytic activity to its ability to increase plasma activator levels (Sawyer et al., 1960), though the mechanism by which it does so is not known. In the present assessment of Complamin as a fibrinolytic agent, factors studied after its administration included plasma fibrinolytic activity, plasminogen and fibrinogen levels, thrombin clotting-time and one-stage prothrombin time, and type and incidence of side-effects.

\section{Materials and Methods}

Complamin was made available by Edinburgh Pharmaceutical Industries as ampoules containing $300 \mathrm{mg}$. in $2 \mathrm{ml}$. and $150-\mathrm{mg}$. tablets. The minimum dosage of the intravenous preparation suggested by the manufacturers is $300 \mathrm{mg}$. t.i.d., and of the tablets 150 mg. t.i.d.

Human fibrinogen was prepared from expired bank plasma by a phosphate buffer method (Biggs and Macfarlane, 1962).

${ }^{131}$ I-tagged fibrinogen was prepared by the method of Clement and McNicol (1959) as modified by McNicol and Douglas (1964). Clottable radioactivity was about $92 \%$. Samples were counted in a well-type scintillation counter with a pulse-height analyser.

Bovine Fibrinogen.-A purified bovine preparation supplied by Dr. I. M. Grondahl, Stockholm, was used.

Thrombin.-Parke Davis thrombin topical was used.

Plasminogen.-A preparation of human plasminogen, soluble at neutral $p \mathrm{H}$, was made available by the Kabi Pharmaceutical Company.

Venous blood was collected by clean venepuncture: $9 \mathrm{ml}$. of blood was mixed with $1 \mathrm{ml}$. of $3.8 \%$ sodium citrate in a graduated centrifuge tube.

Euglobulin Lysis Time.-The method of Nilsson and Olow (1962) was used. As suggested by Sherry et al. (1959b), euglobulin lysis times are expressed as units obtained from a double logarithmic plot of lysis time against units of activity. The unit used is that of McNicol et al. (1963), in which a lysis time of 300 minutes is arbitrarily assigned one unit of activity.

Activator assay with plasminogen-enriched ${ }^{131}$ I-tagged clots was performed by the method of Alkjaersig et.al. (1959a) as modified by McNicol and Douglas (1964). In this assay system the test plasma is incubated with plasminogen-enriched ${ }^{131}$ I-labelled plasma clots.
Radioactivity released from the clots during incubation is a measure of the activator content of the test plasma.

Fibrinogen assays were carried out by the method of Ratnoff and Menzie (1951) as modified by Alkjaersig (1960). The method is described by McNicol and Douglas (1964).

Plasminogen assays were carried out by the method of Remmert and Cohen (1949) as modified by Alkjaersig et al. (1959b). The method is described by McNicol and Douglas (1964).

Thrombin clotting-times were performed by the method of Fletcher et al. (1959).

One-stage prothrombin times were carried out as described by Douglas (1962).

Fibrin plate tests were performed as described by Nilsson and Olow (1962).

\section{Results}

Single Intravenous Injections.-Seven subjects (healthy colleagues) were given a single intravenous injection of Complamin, $150 \mathrm{mg}$. diluted in $10 \mathrm{ml}$. of saline being injected over 60 seconds. The response in euglobulin lysis activity is shown in Fig. 1, where it will be seen that five minutes after injection there was a rise in euglobulin lysis activity from a mean preinfusion level of 1.2 units to a mean level of 7.9 units. Euglobulin lysis activity was almost back to normal at 30

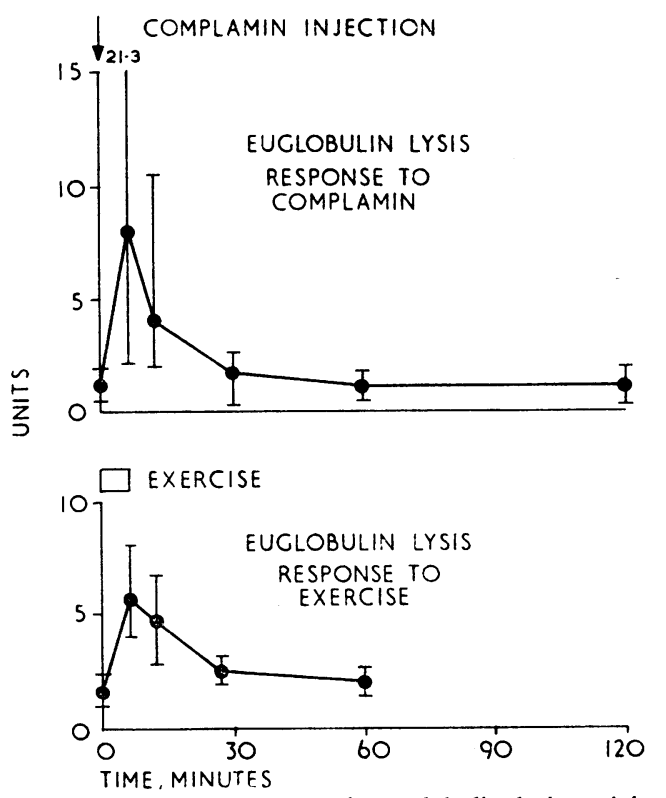

FIG. 1.-Top: Response in euglobulin lysis activity to intravenous injection of $150 \mathrm{mg}$. of Complamin in seven healthy subjects; mean values and ranges are also shown. Bottom: Effect of five minutes'. strenuous exercise on euglobulin lysis activity in the same subjects.

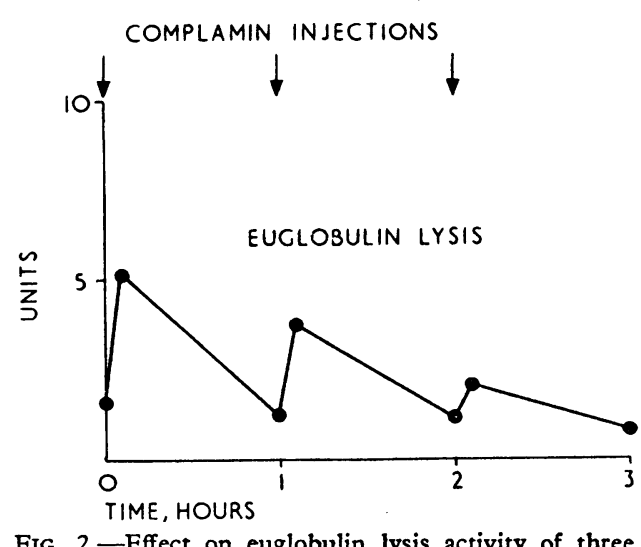

FIG. 2.-Effect on euglobulin lysis activity of three intravenous in jections of $150 \mathrm{mg}$. of Complamin given to a healthy subject at hourly intervals. 
minutes. There was a slight drop in mean fibrinogen and plasminogen levels, but this was not statistically significant and there were no changes in thrombin clotting-times or prothrombin times. The response of the same subjects on another day to exercise (five minutes' skipping with a heavy rubber tube) is also shown in Fig. 1, where a similar pattern of euglobulin lysis activity is seen. The peak rise is somewhat less than that seen with Complamin, but the general pattern of rise and fall of activity is closely parallel. With exercise there were no changes in plasminogen or fibrinogen levels and thrombin and prothrombin times.

Repeated Intravenous Injections. - The response in a healthy colleague to three intravenous injections of $150 \mathrm{mg}$. of Complamin given at hourly intervals is shown in Fig. 2, where it will be seen that there is a progressive reduction in euglobulin lysis activity induced by the second and third injections. Another healthy subject was given a daily intravenous injection of $150 \mathrm{mg}$. of Complamin for five days. The euglobulin lysis response is shown in Fig. 3, where once again a progressive reduction in response is seen.

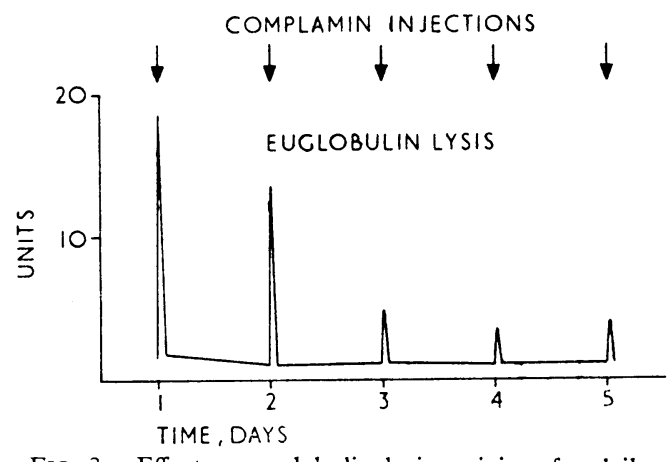

FIG. 3.-Effect on euglobulin lysis activity of a daily intravenous injection of $150 \mathrm{mg}$. of Complamin for five days.

Intravenous Infusions.-An intravenous infusion of Complamin was given to two subjects with very similar results. The effects of the infusion in one of the subjects are shown in Fig. 4. Over the first 30 minutes $150 \mathrm{mg}$. was infused, over the next 30 minutes $300 \mathrm{mg}$., and over the last 30 minutes $600 \mathrm{mg}$. As detected by the radioactive clot method, euglobulin lysis, and fibrin plate test, infusion produced a marked but transient increase in plasminogen activator levels in the plasma, which fell off rapidly despite progressive doubling of the infusion

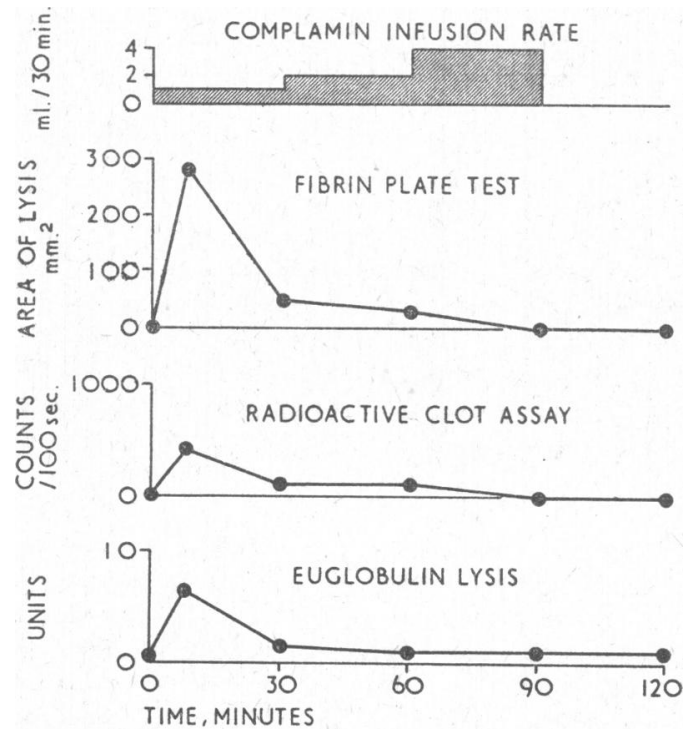

FIG. 4.-Effect on plasma as assessed by the radioactive clot system. the fibrin plate test, and euglobulin lysis activity of intravenous infusion of Complamin at a progressively increased rate $(1 \mathrm{ml} .=150 \mathrm{mg}$.). rate. There were no significant changes in plasminogen, fibrinogen, thrombin time, or prothrombin time. In another subject $150 \mathrm{mg}$. of Complamin infused over one hour produced a rise in euglobulin lysis activity from 0.8 unit before infusion to 1.6 units after 30 minutes and 1.2 units at the end of the infusion. One hour later the euglobulin lysis activity was 1.1 units.

Oral Administration.-Complamin tablets were given to four normal adult male subjects. One subject was given two tablets only; the others were given two tablets on one day and four tablets several days later. The results are shown in the Table, where it will be seen that with two tablets there was a minor increase in euglobulin lysis activity in Subjects 1 and 3, but no change in Subjects 2 and 4 . With four tablets, Subject 2 showed no change, euglobulin lysis activity doubled in Subject 4 , and showed a significant increase in Subject 3. With two tablets, where a rise in euglobulin lysis activity was seen, it was noted in the 30-minute samples but had subsided at 60 minutes. With four tablets the effect seemed to be somewhat longerlasting, being noted in both Subjects 3 and 4 at 120 minutes.

Effect on Euglobulin Lysis Activity of Ingestion of Complamin Tablets in Different Subjects

\begin{tabular}{|c|c|c|c|c|c|c|c|}
\hline \multirow{2}{*}{$\begin{array}{l}\text { Subject } \\
\text { No. }\end{array}$} & \multirow{2}{*}{$\begin{array}{l}\text { No. of } \\
\text { Tablets }\end{array}$} & \multicolumn{6}{|c|}{$\begin{array}{c}\text { Euglobulin Lysis (Units) at Varying Times (Minutes) } \\
\text { after Ingestion }\end{array}$} \\
\hline & & 0 & 10 & 30 & 60 & 120 & 240 \\
\hline $\begin{array}{l}1 \\
2 \\
3 \\
4 \\
2 \\
3 \\
4\end{array}$ & $\begin{array}{l}2 \\
2 \\
2 \\
2 \\
4 \\
4 \\
4\end{array}$ & $\begin{array}{l}2.0 \\
1.1 \\
1.0 \\
1.5 \\
1.4 \\
1.1 \\
1.3\end{array}$ & $\begin{array}{l}\overline{1.2} \\
0.9 \\
1.4 \\
\frac{1.3}{1.4}\end{array}$ & $\begin{array}{l}2.5 \\
1.0 \\
1.3 \\
1.6 \\
1.5 \\
1.4 \\
2.6\end{array}$ & $\begin{array}{l}2.0 \\
0.9 \\
1.0 \\
1.5 \\
1.4 \\
1.5 \\
2.5\end{array}$ & $\begin{array}{l}1.9 \\
1.1 \\
1.2 \\
1.4 \\
1.2 \\
1.9 \\
1.5\end{array}$ & $\begin{array}{l}2 \cdot 1 \\
1 \cdot 2 \\
1 \cdot 1 \\
1 \cdot 4 \\
1 \cdot 3 \\
1 \cdot 3 \\
1 \cdot 4\end{array}$ \\
\hline
\end{tabular}

\section{Complamin and Exercise}

In order to study the interrelationships between the fibrinolytic responses to Complamin and to exercise, two healthy subjects participated in the following experiments, each experiment being carried out separated from the others by an interval of several days: (a) Five minutes' exercise, followed in 30 minutes by five minutes' exercise ; $(b)$ Five minutes' exercise, followed in 30 minutes by $150 \mathrm{mg}$. of Complamin intravenously ; (c) 150 $\mathrm{mg}$. of Complamin intravenously, followed in 30 minutes by five minutes' exercise ; (d) $150 \mathrm{mg}$. of Complamin intravenously,

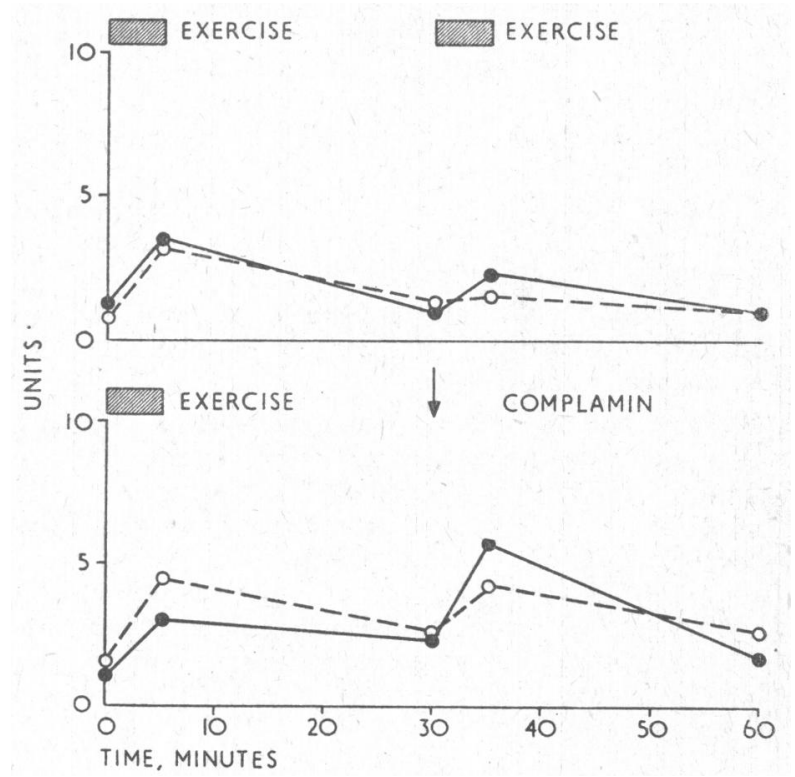

FIG. 5.-Top: Effect, in two subjects, on the euglobulin lysis activity of five minutes' exercise followed in 30 minutes by a further five minutes' exercise. Bottom: Effect on another. occasion of five minutes' exercise followed in 30 minutes by $150 \mathrm{mg}$. of Complamin intravenously. 
followed in 30 minutes by $150 \mathrm{mg}$. of Complamin intravenously. Response of euglobulin lysis activity is shown for experiments $(a)$ and $(b)$ in Fig. 5 and for experiments $(c)$ and $(d)$ in Fig 6.

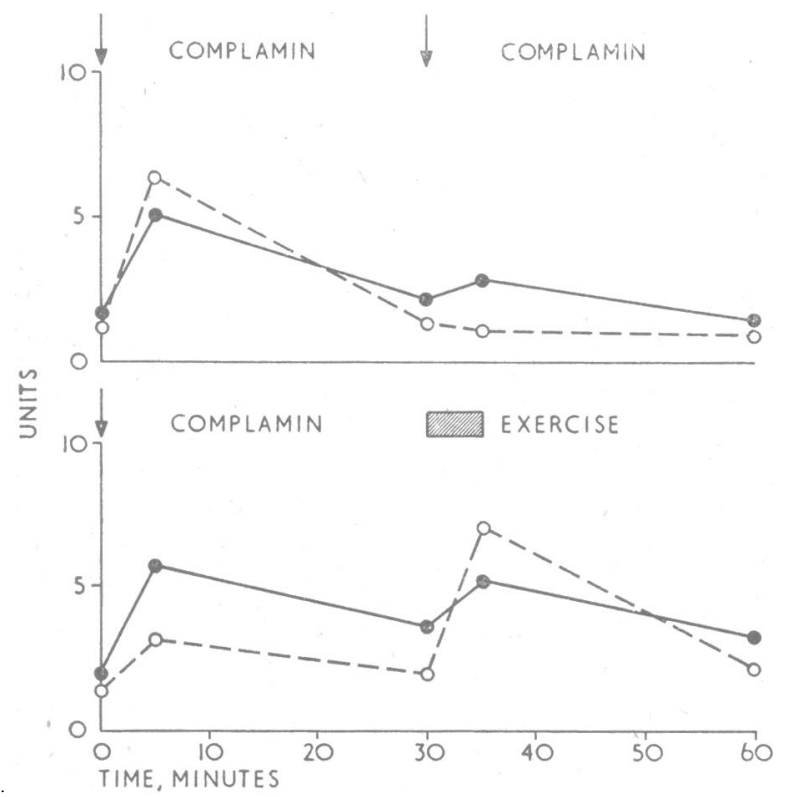

FIg. 6.-Same two subjects as in Fig. 5. Top: Effect on euglobulin lysis activity of $150 \mathrm{mg}$. of Complamin injected intravenously, followed in 30 minutes by a second intravenous injection of $150 \mathrm{mg}$ of Complamin lnjectlon another occasion of $150 \mathrm{mg}$. of Complamin injected intra-
venously, followed in $30 \mathrm{minutes}$ by five minutes' exercise.

It will be seen that for both subjects, if exercise is followed by exercise, or Complamin by Complamin, the second stimulus provokes a much reduced euglobulin lysis response, whereas if exercise is followed by Complamin, or Complamin by exercise, a full response is observed. It may be that the reduced response on the second occasion to repeated exercise is due to fatigue and a less vigorous performance of the exercise.

\section{Side-effects}

Three main side-effects were encountered:

1. Flushing of the skin, with a sensation of tingling and discomfort in the skin. The flush, which was observed in all subjects given Complamin by intravenous injection, appeared gradually a few minutes after injection and persisted for 30 to 45 minutes. There was no correlation between the duration or intensity of the flush and the degree of fibrinolytic response. The three subjects given an intravenous infusion did not show a flush. After oral administration of Complamin all subjects had a flush with four tablets, but only Subjects 3 and 4 had a flush with two tablets.

2. One subject consistently experienced transient upper abdominal pain two to three minutes after Complamin injection, and another had rectal pain.

3. Hypotension. Three subjects had marked hypotension after Complamin injection. In one subject, given $150 \mathrm{mg}$. of Complamin in the prone position, blood-pressure fell from $112 / 65$ to $95 / 5510$ minutes after injection; in the second subject, given $150 \mathrm{mg}$. of Complamin when seated, the fall was from $118 / 72$ to $65 / 40$ at 10 minutes ; and in the third, given $150 \mathrm{mg}$. of Complamin when prone, blood-pressure dropped from $140 / 85$ before injection to $75 / 55$ after injection. All subjects showing hypotension had a marked flush.

\section{Discussion}

The results presented in this communication demonstrate that Complamin, like nicotinic acid, when given rapidly intra- venously, produces a marked but transient rise in plasma fibrinolytic activity, comparable in duration (not more than 30 minutes) and intensity to that produced by five minutes' strenuous exercise. The fibrinolytic response falls off rapidly with injections repeated at hourly intervals and at 24-hour intervals. The response was also transient when Complamin was given by intravenous infusion, contrary to the observations of Amery et al. (1962), who found a response for an hour or more when Complamin was given in this way. Given by mouth, Complamin either, in some subjects, failed to produce a fibrinolytic response or, in other subjects, produced trivial effect.

The mechanism by which Complamin produces increased levels of fibrinolytic activity in the circulation is not clear, though it is shared by some other vaso-active drugs-for example, adrenaline and Pitressin (von Kaulla, 1963). Histamine, however, which produces vasodilatation, does not cause increased plasma fibrinolytic activity (Weiner et al., 1959), and there is no correlation between the intensity of the flush produced by Complamin and the degree of fibrinolytic activity induced. Kwaan et al. (1958) have shown that fibrinolytic moieties can be released from the vessel wall in response to paravenous injection of adrenaline, serotonin, and acetylcholine in the same and in the opposite arm, and it may be that a neural pathway stimulated by Complamin and other substances can bring about plasminogen activator release into the circulation.

The short duration of the fibrinolytic response to Complamin is due in part to the rapid clearance of fibrinolytic activator from the circulation by the liver (Fletcher et al., 1964) and also to the refractory state to the drug which develops. The experiments in which Complamin given after exercise, or exercise taken after Complamin, produces a full fibrinolytic response, suggest that the failure to produce a repeated or sustained response to Complamin is not due to exhaustion of the body's fibrinolytic potential but to resistance to the action of the drug. The experiments suggest that it may be possible to find a drug to produce a sustained release of plasminogen activator into the circulation.

The failure in the present experiments to see significant changes in plasminogen, fibrinogen, thrombin clotting-time, and one-stage prothrombin time is probably due to the short duration of the increase in activator levels produced in healthy subjects by Complamin injection. Fletcher et al. (1964) gave nicotinic acid by intravenous injection to patients with cirrhosis of the liver in whom there was impaired clearance of plasminogen activator from the circulation, and hence a persistence of high levels of activator for much longer than in normal subjects, and found evidence of hyperplasminaemia, including a drop in plasminogen and fibrinogen values and a rise in the thrombin clotting-time and one-stage prothrombin time.

\section{Side-effects}

While the flushing of the skin experienced by all subjects given Complamin by intravenous injection, and the abdominal or rectal pain experienced by two other subjects, were uncomfortable or unpleasant, the hypotension produced in some subjects by intravenous injection of half the manufacturer's suggested minimum dose was a potentially dangerous response which in our opinion represents an absolute contraindication to the use of the drug in patients with myocardial or cerebral infarction.

In thrombolytic therapy with streptokinase, where intense fibrinolytic activity-for example, activity at least as great as the maximum response seen to Complamin in any subject-can readily be achieved and maintained, treatment may need to be continued for 48 hours or more (Johnson and McCarty, 1959 ; Verstraete et al., 1963 ; Douglas and McNicol, 1964) to remove thrombus and restore vascular patency. Despite the manufacturer's claims, it would therefore appear most improbable that Complamin would be of benefit in the treatment of acute 
thromboembolic occlusive vascular disease, for which in any event side-effects render it unsafe. On the other hand, as the present studies demonstrate, Complamin clearly does not exploit the full fibrinolytic potential of the body, and a search for further modifications of nicotinic acid or other pharmacological substances which would produce a sustained fibrinolytic response would seem worth while. In particular, a non-toxic substance administered by mouth might, by long-term administration to increase physiological fibrinolytic activity, have an important role in the prophylaxis of vascular thrombosis.

\section{Summary}

Complamin, a nicotinic-acid/theophylline ester, produces brisk but transient fibrinolytic activity when injected or infused intravenously. A refractory state soon appears, in which patients do not respond to further therapy. However, prior fibrinolytic response to exercise dose not reduce the response to Complamin, nor is the fibrinolytic response to exercise reduced when exercise follows Complamin. It would therefore appear that resistance to repeated injections of Complamin is not due to exhaustion of the body's fibrinolytic potential. Side-effects of Complamin include flushing of the skin, abdominal pain, and hypotension, which may be severe. Because of the transient nature of the fibrinolytic response and the danger of hypotension, Complamin does not appear to have a place as a therapeutic agent in the treatment of thromboembolic occlusive vascular disease.

We are grateful to Professor E. M. McGirr for his interest in this work, and to the colleagues who volunteered to participate in the studies. Miss S. B. Gale provided scientific assistance and the Medical Research Council gave financial support. Complamin was made available by Dr. Jouhar, of Edinburgh Pharmaceutical Industries.

\section{REFERENCES}

Alkjaersig, N. (1960). In N.I.H. Conference on Thrombolytic Agents, edited by H. R. Roberts, and D. Geraty, p. 136. Chapel Hill. edited by H. R. Roberts, and D. Geraty, $\mathrm{p}_{\dot{j}}$ clin. Invest., 38, 1086. - Fletcher, A. P., and Sherry, S. (1959a). 7. clin.

Amery, A., Vermylen, J., Maes, H., and Verstraete, M. (1962). Nouv. Rev. franç. Hémat., 2, 70.

Biggs, R., and Macfarlane, R. G. (1962). Human Blood Coagulation and Its Disorders, 3rd ed., p. 372. Blackwell, Oxford.

Clement, W. E., and McNicol, G. P. (1959). F. clin. Path., 12, 544.

Douglas, A. S. (1962). Anticoagulant Therapy, p. 279. Blackwell, Oxford.

and McNicol, G. P. (1964). Brit. med. Bull., 20, 228.

Fletcher, A. P., Alkjaersig, N., and Sherry, S. (1959). F. clin. Invest., 38 1096.

Biederman, O., Moore, D., Alkjaersig, N., and Sherry S. (1964). Ibid., 43, 681.

Johnson, A. J., and McCarty, W. R. (1959). Ibid., 38, 1627.

von Kaulla, K. N. (1963). Chemistry of Thrombolysis: Human Fibrinolytic Enzymes, p. 244. Thomas, Springfield.

Kwaan, H. C., Lo, R., and MacFadzean, A. J. S. (1958). Clin. Sci., 17, 361.

McNicol, G. P., and Douglas, A. S. (1964). In Recent Advances in Clinical Pathology, series IV, edited by S. C. Dyke, p. 187. Churchill, London.

Gale, S. B., and Douglas, A. S. (1963). Brit. med. 7., 1, 909

Meneghini, P., and Piccinini, F. (1958). Arch. E. Maragliano Pat. Clin. 14, 69 .

Nilsson, I. M., and Olow, B. (1962). Acta chir. scand., 123, 247.

Ratnoff, O. D., and Menzie, C. (1951). F. Lab. clin. Med., 37, 316

Remmert, L. F., and Cohen, P. P. (1949). F. biol. Chem., 181, 431.

Sawyer, W. D., Fletcher, A. P., Alkjaersig, N., and Sherry, S. (1960). F. clin. Invest., 39, 426

Sherry, S., Fletcher, A. P., and Alkjaersig, N. (1959a). Physiol. Rev. 39, 343 .

Lindemeyer R. I Fletcher, A. P., and Alkjaersig, N. (1959b). clin. Invest., $38,810$.

Verstraete, M., Amery, A., and Vermylen, J. (1963). Brit. med. F., 1,

Weiner, M., de Crinis, K., Redisch, W., and Steele, J. M. (1959). Circulation, 19, 845 .

Redisch, W., and Steele, J. M. (1958). Proc. Soc. exp. Biol. (N.Y.), 98, 755 .

\title{
Effect of Obesity and of Starvation on Insulin Activity
}

\author{
N. SAMAAN, ${ }^{*} \ddagger$ M.B., B.CH., M.R.C.P.ED. ; J. BROWN, $\dagger$ M.B., B.S., B.SC., D.C.P. ; \\ RUSSELL FRASER,* M.D., F.R.C.P., D.P.M.; I. TRAYNER,* M.B., B.S.
}

Brit. med. F., 1965, 1, 1153-1156

It has long been established that obesity may lead to development of diabetes mellitus, and also that weight reduction often leads to a complete remission of this diabetes mellitus (Newburg and Conn, 1939). However, since diabetes in the obese, as well as other diabetic states, can be corrected by insulin, its basis may well be a relative insulin deficiency, whether from lack of production, excessive utilization, or both. Yet in obese diabetics measurement of the serum insulin-like activity usually shows normal or high levels, which at first sight does not seem to fit this hypothesis (Bornstein and Lawrence, 1951 ; Vallance-Owen et al., 1955 ; Seltzer and Smith, 1959; Steinke et al., 1961a, 1962 ; Samaan et al., 1962b). The present studies were therefore undertaken to try to clarify some of the factors affecting insulin activity in obesity.

In obese mice have been found hypertrophied pancreatic islet beta-cells, raised levels of serum insulin-like activity, both by bioassay and immunoassay, and also decreased tissue responsiveness to insulin; these features being found both in genetic obesity and in that induced by hypathalamic lesions (Christophe

* Department of Medicine, Postgraduate Medical School of London. † Department of Chemical Pathology, Postgraduate Medical School of London.

‡ Present address : Department of Medicine, Lakeside Hospital, Cleveland, Ohio, U.S.A. et al., 1959 ; Hales and Kennedy, 1963 ; Sneyd, 1964). And, correspondingly, in obese humans there has also been found evidence both of hyperinsulinism and of insulin resistance ; for the former from serum insulin assays (Karam et al., 1963; Samaan and Fraser, 1963) and from forearm metabolic studies (Rabinowitz and Zierler, 1962), and for the latter from intravenous-insulin-tolerance tests, both by standard techniques (Fraser et al., 1962) and by localized forearm techniques (Rabinowitz and Zierler, 1962).

As Randle et al. (1963) have suggested, the diabetic tendency in obesity, as well as that in starvation and Cushing's syndrome, may arise from impairment of the action of insulin by the increased concentration of fat metabolites prevailing in these states. Certainly there are raised levels of serum free fatty acids in obesity (Gordon, 1960 ; Corvilain et al., 1961 ; Opie and Walfish, 1963), as well as in starvation where ketosis also prevails (Kekwick et al., 1959) ; and the action of insulin on tissue is known to be impaired either by raised free fatty acid levels (Williamson and Krebs, 1961; Shipp et al., 1961), or by increased concentrations of ketone bodies (Felts et al., 1964). But other factors must also be involved; since starvation, while indeed inducing in normal subjects a mild diabetes, can in the established diabetic considerably lessen his hyperglycaemia (Gephart et al., 1917 ; Allen, 1922). Presumably starvation 